\title{
A class of retarded Volterra-Fredholm type integral inequalities on time scales and their applications
}

Haidong Liu*

\section{"Correspondence:} tomlhd983@163.com School of Mathematical Sciences, Qufu Normal University, Qufu, 273165, P.R. China

\section{Springer}

\begin{abstract}
In this paper, we study some new retarded Volterra-Fredholm type integral inequalities on time scales, which provide explicit bounds on unknown functions. These inequalities generalize and extend some known inequalities and can be used as tools in the qualitative theory of certain classes of retarded dynamic equations on time scales. Some applications are also presented to illustrate the usefulness of our results.
\end{abstract}

Keywords: time scale; retarded integral inequality; Volterra-Fredholm type

\section{Introduction}

In 1988, Hilger introduced the theory of time scales in order to unify and extend the difference and differential calculus in a consistent way (see [1]). Since then, more and more researchers are getting involved in this fast-growing field, for example, [2-10] and the references therein. Among various aspects of the theory, we notice that dynamic inequalities on time scales is an object of long standing interest [11-29]. However, to the best of our knowledge, there are few results dealing with Volterra-Fredholm type integral inequalities on time scales. Recent results in this direction include the works of Gu and Meng [16] and Meng and Shao [20].

The purpose of this paper is to investigate some new retarded Volterra-Fredholm type integral inequalities on time scales, which not only generalize and extend the results of $[16,20]$ and some known integral inequalities but also provide a handy and effective tool for the study of qualitative properties of solutions of some complicated Volterra-Fredholm type dynamic equations.

The paper is organized as follows. In Section 2, some necessary definition and lemmas are presented. In Section 3, some new retarded Volterra-Fredholm type integral inequalities on time scales are investigated. Finally, Section 4 is devoted to applying our results to a retarded Volterra-Fredholm type dynamic integral equation on time scales.

\section{Preliminaries}

Throughout this paper, knowledge and understanding of time scales and time scale notation is assumed. For an excellent introduction to the calculus on time scales, we refer the reader to [30] and [31].

(c) The Author(s) 2017. This article is distributed under the terms of the Creative Commons Attribution 4.0 International License (http://creativecommons.org/licenses/by/4.0/), which permits unrestricted use, distribution, and reproduction in any medium, provided you give appropriate credit to the original author(s) and the source, provide a link to the Creative Commons license, and indicate if changes were made. 
List of abbreviations. In what follows, we always assume that $\mathbb{R}$ denotes the set of real numbers, $\mathbb{R}_{+}=[0, \infty), \mathbb{Z}$ denotes the set of integers, $\mathbb{T}$ is an arbitrary time scale (nonempty closed subset of $\mathbb{R}), \mathcal{R}$ denotes the set of all regressive and rd-continuous functions, $\mathcal{R}^{+}=$ $\{p \in \mathcal{R}: 1+\mu(t) p(t)>0$ for all $t \in \mathbb{T}\}$ and $I=\left[t_{0}, T\right] \cap \mathbb{T}^{\kappa}$, where $t_{0} \in \mathbb{T}^{\kappa}, T \in \mathbb{T}^{\kappa}, T>$ $t_{0}$. The set $\mathbb{T}^{\kappa}$ is defined as follows: If $\mathbb{T}$ has a maximum $m$ and $m$ is left-scattered, then $\mathbb{T}^{\kappa}=\mathbb{T}-\{m\}$. Otherwise $\mathbb{T}^{\kappa}=\mathbb{T}$. The graininess function $\mu: \mathbb{T} \rightarrow[0, \infty)$ is defined by $\mu(t):=\sigma(t)-t$, the forward jump operator $\sigma: \mathbb{T} \rightarrow \mathbb{T}$ by $\sigma(t):=\inf \{s \in \mathbb{T}: s>t\}$, and the "circle plus" addition $\oplus$ is defined by $(p \oplus q)(t):=p(t)+q(t)+\mu(t) p(t) q(t)$ for all $t \in \mathbb{T}^{\kappa}$.

The following lemmas and definition are useful in the proof of the main results of this paper.

Lemma 2.1 ([30, Theorem 1.16]) Assume that $f: \mathbb{T} \rightarrow \mathbb{R}$ is a function and let $t \in \mathbb{T}$. If $f$ is differentiable at $t$, then

$$
f(\sigma(t))=f(t)+\mu(t) f^{\Delta}(t)
$$

Lemma 2.2 ([30, Theorem 1.98]) Assume that $v: \mathbb{T} \rightarrow \mathbb{R}$ is a strictly increasing function and $\widetilde{\mathbb{T}}:=v(\mathbb{T})$ is a time scale. If $f: \mathbb{T} \rightarrow \mathbb{R}$ is an $r d$-continuous function and $v$ is differentiable with $r d$-continuous derivative, then for $a, b \in \mathbb{T}$,

$$
\int_{a}^{b} f(t) v^{\Delta}(t) \Delta t=\int_{v(a)}^{v(b)}\left(f \circ v^{-1}\right)(s) \widetilde{\Delta} s
$$

Lemma 2.3 Let $\alpha: I \rightarrow I$ be a continuous and strictly increasing function such that $\alpha(t) \leq$ $t$, and $\alpha^{\Delta}$ is $r d$-continuous. Assume that $f: I \rightarrow \mathbb{R}$ is an $r d$-continuous function, then

$$
g(t)=\int_{\alpha\left(t_{0}\right)}^{\alpha(t)} f(s) \Delta s, \quad t \in I
$$

implies

$$
g^{\Delta}(t)=f(\alpha(t)) \alpha^{\Delta}(t), \quad t \in I .
$$

Proof From (2.1), we get, for any $t \in I$,

$$
g(t)=\int_{\alpha\left(t_{0}\right)}^{\alpha(t)} f(s) \Delta s=\int_{\alpha\left(t_{0}\right)}^{\alpha(t)} f\left(\alpha\left(\alpha^{-1}(s)\right)\right) \Delta s .
$$

By Lemma 2.2, we obtain

$$
g(t)=\int_{t_{0}}^{t} f(\alpha(s)) \alpha^{\Delta}(s) \Delta s, \quad t \in I
$$

so we get

$$
g^{\Delta}(t)=f(\alpha(t)) \alpha^{\Delta}(t)
$$


Lemma 2.4 ([30, Theorem 1.117]) Suppose that, for each $\varepsilon>0$, there exists a neighborhood $U$ of $t$, independent of $\tau \in\left[t_{0}, \sigma(t)\right]$, such that

$$
\left|w(\sigma(t), \tau)-w(s, \tau)-w_{t}^{\Delta}(t, \tau)(\sigma(t)-s)\right| \leq \varepsilon|\sigma(t)-s|, \quad s \in U
$$

where $w: \mathbb{T} \times \mathbb{T}^{\kappa} \rightarrow \mathbb{R}_{+}$is continuous at $(t, t), t \in \mathbb{T}^{\kappa}$ with $t>t_{0}$, and $w_{t}^{\Delta}(t, \cdot)$ are $r d$ continuous on $\left[t_{0}, \sigma(t)\right]$. Then

$$
g(t):=\int_{t_{0}}^{t} w(t, \tau) \Delta \tau
$$

implies

$$
g^{\Delta}(t)=\int_{t_{0}}^{t} w_{t}^{\Delta}(t, \tau) \Delta \tau+w(\sigma(t), t), \quad t \in \mathbb{T}^{\kappa} .
$$

Lemma 2.5 ([30, Theorem 6.1]) Suppose that $y$ and $f$ are $r d$-continuous functions and $p \in \mathcal{R}^{+}$. Then

$$
y^{\Delta}(t) \leq p(t) y(t)+f(t) \quad \text { for all } t \in \mathbb{T}
$$

implies

$$
y(t) \leq y\left(t_{0}\right) e_{p}\left(t, t_{0}\right)+\int_{t_{0}}^{t} e_{p}(t, \sigma(\tau)) f(\tau) \Delta \tau \quad \text { for all } t \in \mathbb{T} .
$$

Definition 2.1 A function $x: I \rightarrow I$ is said to belong to the class $\Upsilon$ if

(1) $x$ is continuous and strictly increasing, and

(2) $x(t) \leq t$ and $x^{\Delta}$ is rd-continuous.

\section{Main results}

Theorem 3.1 Assume that $\alpha \in \Upsilon$ and $u, a, b, f_{1}, f_{2}, g: I \rightarrow \mathbb{R}_{+}$are $r d$-continuous functions, a is nondecreasing, $\lambda \geq 0$ is a constant, $\alpha^{\Delta}(t) \geq 0, b^{\Delta}(t) \geq 0, \mu(t) A(t)<1$. Suppose that $u$ satisfies

$$
\begin{aligned}
u(t) \leq & a(t)+b(t) \int_{\alpha\left(t_{0}\right)}^{\alpha(t)}\left[f_{1}(s) u(s)+f_{2}(s) \int_{\alpha\left(t_{0}\right)}^{s} g(\tau) u(\tau) \Delta \tau\right] \Delta s \\
& +\lambda b(T) \int_{\alpha\left(t_{0}\right)}^{\alpha(T)}\left[f_{1}(s) u(s)+f_{2}(s) \int_{\alpha\left(t_{0}\right)}^{s} g(\tau) u(\tau) \Delta \tau\right] \Delta s, \quad t \in I .
\end{aligned}
$$

If

$$
\beta:=e_{B \oplus C}\left(T, t_{0}\right)<1+\frac{1}{\lambda}
$$

then

$$
u(t) \leq \frac{a(T)}{\lambda+1-\beta \lambda} e_{B \oplus C}\left(t, t_{0}\right), \quad t \in I,
$$


where we use the convention that $\frac{1}{0}=+\infty$,

$$
\begin{aligned}
& A(t):=b^{\Delta}(t) \int_{\alpha\left(t_{0}\right)}^{\alpha(\sigma(t))}\left[f_{1}(s)+f_{2}(s) \int_{\alpha\left(t_{0}\right)}^{s} g(\tau) \Delta \tau\right] \Delta s, \quad B(t):=\frac{A(t)}{1-\mu(t) A(t)}, \\
& C(t):=b(t)\left[f_{1}(\alpha(t))+f_{2}(\alpha(t)) \int_{\alpha\left(t_{0}\right)}^{\alpha(t)} g(\tau) \Delta \tau\right] \alpha^{\Delta}(t) .
\end{aligned}
$$

\section{Proof Denote}

$$
\begin{aligned}
z(t):= & a(T)+b(t) \int_{\alpha\left(t_{0}\right)}^{\alpha(t)}\left[f_{1}(s) u(s)+f_{2}(s) \int_{\alpha\left(t_{0}\right)}^{s} g(\tau) u(\tau) \Delta \tau\right] \Delta s \\
& +\lambda b(T) \int_{\alpha\left(t_{0}\right)}^{\alpha(T)}\left[f_{1}(s) u(s)+f_{2}(s) \int_{\alpha\left(t_{0}\right)}^{s} g(\tau) u(\tau) \Delta \tau\right] \Delta s, \quad t \in I .
\end{aligned}
$$

Then $z$ is nondecreasing on $I$,

$$
u(t) \leq z(t), \quad t \in I,
$$

and

$$
z\left(t_{0}\right)=a(T)+\lambda b(T) \int_{\alpha\left(t_{0}\right)}^{\alpha(T)}\left[f_{1}(s) u(s)+f_{2}(s) \int_{\alpha\left(t_{0}\right)}^{s} g(\tau) u(\tau) \Delta \tau\right] \Delta s .
$$

From Lemma 2.3 and (3.4)-(3.7), we have

$$
\begin{aligned}
z^{\Delta}(t)= & b^{\Delta}(t) \int_{\alpha\left(t_{0}\right)}^{\alpha(\sigma(t))}\left[f_{1}(s) u(s)+f_{2}(s) \int_{\alpha\left(t_{0}\right)}^{s} g(\tau) u(\tau) \Delta \tau\right] \Delta s \\
& +b(t)\left[f_{1}(\alpha(t)) u(\alpha(t))+f_{2}(\alpha(t)) \int_{\alpha\left(t_{0}\right)}^{\alpha(t)} g(\tau) u(\tau) \Delta \tau\right] \alpha^{\Delta}(t) \\
\leq & b^{\Delta}(t) \int_{\alpha\left(t_{0}\right)}^{\alpha(\sigma(t))}\left[f_{1}(s) z(s)+f_{2}(s) \int_{\alpha\left(t_{0}\right)}^{s} g(\tau) z(\tau) \Delta \tau\right] \Delta s \\
& +b(t)\left[f_{1}(\alpha(t)) z(\alpha(t))+f_{2}(\alpha(t)) \int_{\alpha\left(t_{0}\right)}^{\alpha(t)} g(\tau) z(\tau) \Delta \tau\right] \alpha^{\Delta}(t) \\
\leq & z(\sigma(t)) b^{\Delta}(t) \int_{\alpha\left(t_{0}\right)}^{\alpha(\sigma(t))}\left[f_{1}(s)+f_{2}(s) \int_{\alpha\left(t_{0}\right)}^{s} g(\tau) \Delta \tau\right] \Delta s \\
& +z(t) b(t)\left[f_{1}(\alpha(t))+f_{2}(\alpha(t)) \int_{\alpha\left(t_{0}\right)}^{\alpha(t)} g(\tau) \Delta \tau\right] \alpha^{\Delta}(t) \\
= & A(t) z(\sigma(t))+C(t) z(t), \quad t \in I .
\end{aligned}
$$

Note that from (3.4) we get

$$
A(t)=\frac{B(t)}{1+\mu(t) B(t)},
$$


and from (3.9) we have

$$
\begin{aligned}
z^{\Delta}(t) & \leq \frac{B(t)}{1+\mu(t) B(t)} z(\sigma(t))+C(t) z(t) \\
& =\frac{B(t)}{1+\mu(t) B(t)}\left[z(t)+\mu(t) z^{\Delta}(t)\right]+C(t) z(t),
\end{aligned}
$$

which yields

$$
\frac{1}{1+\mu(t) B(t)} z^{\Delta}(t) \leq\left[\frac{B(t)}{1+\mu(t) B(t)}+C(t)\right] z(t)
$$

i.e.,

$$
\begin{aligned}
z^{\Delta}(t) & \leq[B(t)+(1+\mu(t) B(t)) C(t)] z(t) \\
& =(B \oplus C)(t) z(t) .
\end{aligned}
$$

Note that $z$ is rd-continuous and $B \oplus C \in \mathcal{R}^{+}$, from Lemma 2.5 and (3.10), we obtain

$$
z(t) \leq z\left(t_{0}\right) e_{B \oplus C}\left(t, t_{0}\right), \quad t \in I .
$$

From (3.6) and (3.8), we get

$$
\left(z\left(t_{0}\right)-a(T)\right) \frac{\lambda+1}{\lambda}+a(T)=z(T),
$$

i.e.,

$$
\frac{\lambda+1}{\lambda} z\left(t_{0}\right)-\frac{1}{\lambda} a(T)=z(T) .
$$

From (3.2), (3.11) and (3.12), we have

$$
\frac{\lambda+1}{\lambda} z\left(t_{0}\right)-\frac{1}{\lambda} a(T)=z(T) \leq z\left(t_{0}\right) e_{B \oplus C}\left(T, t_{0}\right)=z\left(t_{0}\right) \beta .
$$

In view of (3.2) and (3.13), we get

$$
z\left(t_{0}\right) \leq \frac{a(T)}{\lambda+1-\beta \lambda}
$$

Substituting (3.14) into (3.11), we obtain

$$
z(t) \leq \frac{a(T)}{\lambda+1-\beta \lambda} e_{B \oplus C}\left(t, t_{0}\right), \quad t \in I .
$$

Noting $u(t) \leq z(t)$, we get the desired inequality (3.3). This completes the proof.

If we let $\lambda=\frac{1}{b(T)}$ in Theorem 3.1, then we obtain the following corollary. 
Corollary 3.1 Assume that $\alpha, u, a, b, f_{1}, f_{2}, g, A, B, C$ are the same as in Theorem 3.1 and $b(T) \neq 0$. Suppose that $u$ satisfies

$$
\begin{aligned}
u(t) \leq & a(t)+b(t) \int_{\alpha\left(t_{0}\right)}^{\alpha(t)}\left[f_{1}(s) u(s)+f_{2}(s) \int_{\alpha\left(t_{0}\right)}^{s} g(\tau) u(\tau) \Delta \tau\right] \Delta s \\
& +\int_{\alpha\left(t_{0}\right)}^{\alpha(T)}\left[f_{1}(s) u(s)+f_{2}(s) \int_{\alpha\left(t_{0}\right)}^{s} g(\tau) u(\tau) \Delta \tau\right] \Delta s, \quad t \in I .
\end{aligned}
$$

If

$$
\beta:=e_{B \oplus C}\left(T, t_{0}\right)<1+b(T)
$$

then

$$
u(t) \leq \frac{a(T) b(T)}{1+b(T)-\beta} e_{B \oplus C}\left(t, t_{0}\right), \quad t \in I .
$$

Remark 3.1 If we take $a(t) \equiv u_{0}, b(t) \equiv 1, \alpha(t)=t$ and $\lambda=1$, then Theorem 3.1 reduces to [16, Theorem 3]. If we take $a(t) \equiv u_{0}, b(t) \equiv 1, \alpha(t)=t, f_{1}(t)=f_{2}(t)$ and $\lambda=1$, then Theorem 3.1 reduces to [20, Theorem 2.3].

Theorem 3.2 Assume that $\alpha, u, a, b, \lambda$ are the same as in Theorem 3.1 and $\mu(t) \tilde{A}(t)<1$. Let $v(t, s)$ and $w(t, s)$ be defined as in Lemma 2.3 such that $v_{t}^{\Delta}(t, s) \geq 0, w_{t}^{\Delta}(t, s) \geq 0$ for $t \geq s$ and (2.3) holds. Suppose that $u$ satisfies

$$
\begin{aligned}
u(t) \leq & a(t)+b(t) \int_{t_{0}}^{t}\left[v(t, s) u(s)+w(t, s) \int_{\alpha\left(t_{0}\right)}^{\alpha(s)} g(\tau) u(\tau) \Delta \tau\right] \Delta s \\
& +\lambda b(T) \int_{t_{0}}^{T}\left[v(T, s) u(s)+w(T, s) \int_{\alpha\left(t_{0}\right)}^{\alpha(s)} g(\tau) u(\tau) \Delta \tau\right] \Delta s, \quad t \in I .
\end{aligned}
$$

If

$$
\beta:=e_{\widetilde{B} \oplus \widetilde{C}}\left(T, t_{0}\right)<1+\frac{1}{\lambda},
$$

then

$$
u(t) \leq \frac{a(T)}{\lambda+1-\beta \lambda} e_{\widetilde{B} \oplus \widetilde{C}}\left(t, t_{0}\right), \quad t \in I,
$$

where

$$
\begin{aligned}
\widetilde{A}(t):= & b^{\Delta}(t) \int_{t_{0}}^{\sigma(t)}\left[v(\sigma(t), s)+w(\sigma(t), s) \int_{\alpha\left(t_{0}\right)}^{\alpha(s)} g(\tau) \Delta \tau\right] \Delta s, \\
\widetilde{B}(t):= & \frac{\widetilde{A}(t)}{1-\mu(t) \widetilde{A}(t)}, \\
\widetilde{C}(t):= & b(t)\left[v(\sigma(t), t)+w(\sigma(t), t) \int_{\alpha\left(t_{0}\right)}^{\alpha(t)} g(\tau) \Delta \tau\right. \\
& \left.+\int_{t_{0}}^{t}\left[v_{t}^{\Delta}(t, s)+w_{t}^{\Delta}(t, s) \int_{\alpha\left(t_{0}\right)}^{\alpha(s)} g(\tau) \Delta \tau\right] \Delta s\right] .
\end{aligned}
$$




\section{Proof Denote}

$$
\begin{aligned}
z(t):= & a(T)+b(t) \int_{t_{0}}^{t}\left[v(t, s) u(s)+w(t, s) \int_{\alpha\left(t_{0}\right)}^{\alpha(s)} g(\tau) u(\tau) \Delta \tau\right] \Delta s \\
& +\lambda b(T) \int_{t_{0}}^{T}\left[v(t, T) u(s)+w(T, s) \int_{\alpha\left(t_{0}\right)}^{\alpha(s)} g(\tau) u(\tau) \Delta \tau\right] \Delta s, \quad t \in I .
\end{aligned}
$$

Then $z$ is nondecreasing on $I$,

$$
u(t) \leq z(t), \quad t \in I,
$$

and

$$
z\left(t_{0}\right)=a(T)+\lambda b(T) \int_{t_{0}}^{T}\left[v(T, s) u(s)+w(T, s) \int_{\alpha\left(t_{0}\right)}^{\alpha(s)} g(\tau) u(\tau) \Delta \tau\right] \Delta s .
$$

From Lemma 2.3 and (3.15)-(3.21), we have

$$
\begin{aligned}
z^{\Delta}(t)= & b^{\Delta}(t) \int_{t_{0}}^{\sigma(t)}\left[v(\sigma(t), s) u(s)+w(\sigma(t), s) \int_{\alpha\left(t_{0}\right)}^{\alpha(s)} g(\tau) u(\tau) \Delta \tau\right] \Delta s \\
& +b(t)\left[v(\sigma(t), t) u(t)+w(\sigma(t), t) \int_{\alpha\left(t_{0}\right)}^{\alpha(t)} g(\tau) u(\tau) \Delta \tau\right. \\
& \left.+\int_{t_{0}}^{t}\left[v_{t}^{\Delta}(t, s) u(s)+w_{t}^{\Delta}(t, s) \int_{\alpha\left(t_{0}\right)}^{\alpha(s)} g(\tau) u(\tau) \Delta \tau\right] \Delta s\right] \\
\leq & b^{\Delta}(t) \int_{t_{0}}^{\sigma(t)}\left[v(\sigma(t), s) z(s)+w(\sigma(t), s) \int_{\alpha\left(t_{0}\right)}^{\alpha(s)} g(\tau) z(\tau) \Delta \tau\right] \Delta s \\
& +b(t)\left[v(\sigma(t), t) z(t)+w(\sigma(t), t) \int_{\alpha\left(t_{0}\right)}^{\alpha(t)} g(\tau) z(\tau) \Delta \tau\right. \\
& \left.+\int_{t_{0}}^{t}\left[v_{t}^{\Delta}(t, s) z(s)+w_{t}^{\Delta}(t, s) \int_{\alpha\left(t_{0}\right)}^{\alpha(s)} g(\tau) z(\tau) \Delta \tau\right] \Delta s\right] \\
\leq & z(\sigma(t)) b^{\Delta}(t) \int_{t_{0}}^{\sigma(t)}\left[v(\sigma(t), s)+w(\sigma(t), s) \int_{\alpha\left(t_{0}\right)}^{\alpha(s)} g(\tau) \Delta \tau\right] \Delta s \\
& +z(t) b(t)\left[v(\sigma(t), t)+w(\sigma(t), t) \int_{\alpha\left(t_{0}\right)}^{\alpha(t)} g(\tau) \Delta \tau\right. \\
& \left.+\int_{t_{0}}^{t}\left[v_{t}^{\Delta}(t, s)+w_{t}^{\Delta}(t, s) \int_{\alpha\left(t_{0}\right)}^{\alpha(s)} g(\tau) \Delta \tau\right] \Delta s\right] \\
= & \widetilde{A}(t) z(\sigma(t))+\widetilde{C}(t) z(t), \quad t \in I .
\end{aligned}
$$

Similar to the proof of Theorem 3.1, we get (3.17). This completes the proof.

If we let $\lambda=\frac{1}{b(T)}$ in Theorem 3.2, then we obtain the following corollary. 
Corollary 3.2 Assume that $u, a, b, v, w, \widetilde{A}, \widetilde{B}, \widetilde{C}$ are the same as in Theorem 3.2 and $b(T) \neq 0$. Suppose that $u$ satisfies

$$
\begin{aligned}
u(t) \leq & a(t)+b(t) \int_{t_{0}}^{t}\left[v(t, s) u(s)+w(t, s) \int_{\alpha\left(t_{0}\right)}^{\alpha(s)} g(\tau) u(\tau) \Delta \tau\right] \Delta s \\
& +\int_{t_{0}}^{T}\left[v(T, s) u(s)+w(T, s) \int_{\alpha\left(t_{0}\right)}^{\alpha(s)} g(\tau) u(\tau) \Delta \tau\right] \Delta s, \quad t \in I .
\end{aligned}
$$

If

$$
\beta:=e_{\widetilde{B} \oplus \widetilde{C}}\left(T, t_{0}\right)<1+b(T),
$$

then

$$
u(t) \leq \frac{a(T)}{\lambda+1-\beta \lambda} e_{\widetilde{B} \oplus \widetilde{C}}\left(t, t_{0}\right), \quad t \in I .
$$

Theorem 3.3 Assume that $u, a, f, \lambda$ are the same as in Theorem 3.1. Let $g(t, s)$ be defined as in Lemma 2.4 such that $g_{t}^{\Delta}(t, s) \geq 0$ for $t \geq s$ and (2.3) holds. Suppose that $u$ satisfies

$$
\begin{aligned}
u(t) \leq & a(t)+\int_{t_{0}}^{t} f(s)\left[u(s)+\int_{t_{0}}^{s} g(s, \tau) u(\tau) \Delta \tau\right] \Delta s \\
& +\lambda \int_{t_{0}}^{T} f(s)\left[u(s)+\int_{t_{0}}^{s} g(s, \tau) u(\tau) \Delta \tau\right] \Delta s, \quad t \in I .
\end{aligned}
$$

If

$$
\beta:=\int_{t_{0}}^{T} f(s) e_{A}\left(s, t_{0}\right) \Delta s<\frac{1}{\lambda},
$$

then

$$
u(t) \leq \frac{a(T)}{1-\beta \lambda}\left[1+\int_{t_{0}}^{t} f(s) e_{A}\left(s, t_{0}\right) \Delta s\right], \quad t \in I,
$$

where

$$
A(t):=f(t)+g(\sigma(t), t)+\int_{t_{0}}^{t} g_{t}^{\Delta}(t, \tau) \Delta \tau .
$$

Proof Denote

$$
\begin{aligned}
z(t):= & a(T)+\int_{t_{0}}^{t} f(s)\left[u(s)+\int_{t_{0}}^{s} g(s, \tau) u(\tau) \Delta \tau\right] \Delta s \\
& +\lambda \int_{t_{0}}^{T} f(s)\left[u(s)+\int_{t_{0}}^{s} g(s, \tau) u(\tau) \Delta \tau\right] \Delta s, \quad t \in I .
\end{aligned}
$$

Then $z$ is nondecreasing on $I$,

$$
u(t) \leq z(t), \quad t \in I,
$$


and

$$
z\left(t_{0}\right)=a(T)+\lambda \int_{t_{0}}^{T} f(s)\left[u(s)+\int_{t_{0}}^{s} g(s, \tau) u(\tau) \Delta \tau\right] \Delta s .
$$

From Lemma 2.4 and (3.26)-(3.29), we get

$$
\begin{aligned}
z^{\Delta}(t) & =f(t) u(t)+f(t) \int_{t_{0}}^{t} g(t, \tau) u(\tau) \Delta \tau \\
& \leq f(t)\left[z(t)+\int_{t_{0}}^{t} g(t, \tau) z(\tau) \Delta \tau\right], \quad t \in I .
\end{aligned}
$$

Let

$$
V(t)=z(t)+\int_{t_{0}}^{t} g(t, \tau) z(\tau) \Delta \tau, \quad t \in I .
$$

Obviously,

$$
V\left(t_{0}\right)=z\left(t_{0}\right), \quad z(t) \leq V(t), \quad z^{\Delta}(t) \leq f(t) V(t) .
$$

From Lemma 2.4, (3.27) and (3.32), we obtain

$$
\begin{aligned}
V^{\Delta}(t) & =z^{\Delta}(t)+g(\sigma(t), t) z(t)+\int_{t_{0}}^{t} g_{t}^{\Delta}(t, \tau) z(\tau) \Delta \tau \\
& \leq\left[f(t)+g(\sigma(t), t)+\int_{t_{0}}^{t} g_{t}^{\Delta}(t, \tau) \Delta \tau\right] V(t) \\
& =A(t) V(t), \quad t \in I .
\end{aligned}
$$

It is easy to see that $A \in \mathcal{R}^{+}$. Therefore, from Lemma 2.5 and the above inequality, we have

$$
V(t) \leq V\left(t_{0}\right) e_{A}\left(t, t_{0}\right)=z\left(t_{0}\right) e_{A}\left(t, t_{0}\right), \quad t \in I .
$$

Combining (3.33) and (3.34), we get

$$
z^{\Delta}(t) \leq f(t) z\left(t_{0}\right) e_{A}\left(t, t_{0}\right)
$$

Setting $t=\tau$ in (3.35), integrating it from $t_{0}$ to $t$, we easily obtain

$$
z(t) \leq z\left(t_{0}\right)+z\left(t_{0}\right) \int_{t_{0}}^{t} f(s) e_{A}\left(s, t_{0}\right) \Delta s
$$

From (3.28) and (3.30), we have

$$
\left(z\left(t_{0}\right)-a(T)\right) \frac{\lambda+1}{\lambda}+a(T)=z(T),
$$

i.e.,

$$
\frac{\lambda+1}{\lambda} z\left(t_{0}\right)-\frac{1}{\lambda} a(T)=z(T) .
$$


From (3.25), (3.36) and (3.37), we have

$$
\frac{\lambda+1}{\lambda} z\left(t_{0}\right)-\frac{1}{\lambda} a(T)=z(T) \leq z\left(t_{0}\right)+z\left(t_{0}\right) \int_{t_{0}}^{T} f(s) e_{A}\left(s, t_{0}\right) \Delta s=z\left(t_{0}\right)+z\left(t_{0}\right) \beta .
$$

In view of (3.25), we get

$$
z\left(t_{0}\right) \leq \frac{a(T)}{1-\beta \lambda}
$$

Substituting (3.38) into (3.36), we have

$$
z(t) \leq \frac{a(T)}{1-\beta \lambda}\left[1+\int_{t_{0}}^{t} f(s) e_{A}\left(s, t_{0}\right) \Delta s\right]
$$

Noting $u(t) \leq z(t)$, we get the desired inequality (3.26). This completes the proof.

If we let $\lambda=1$ in Theorem 3.3, then we obtain the following corollary.

Corollary 3.3 Assume that $u, a, f, g$ and $A$ are the same as in Theorem 3.3. Suppose that u satisfies

$$
\begin{aligned}
u(t) \leq & a(t)+\int_{t_{0}}^{t} f(s)\left[u(s)+\int_{t_{0}}^{s} g(s, \tau) u(\tau) \Delta \tau\right] \Delta s \\
& +\int_{t_{0}}^{T} f(s)\left[u(s)+\int_{t_{0}}^{s} g(s, \tau) u(\tau) \Delta \tau\right] \Delta s, \quad t \in I .
\end{aligned}
$$

If

$$
\beta:=\int_{t_{0}}^{T} f(s) e_{A}\left(s, t_{0}\right) \Delta s<1
$$

then

$$
u(t) \leq \frac{a(T)}{1-\beta}\left[1+\int_{t_{0}}^{t} f(s) e_{A}\left(s, t_{0}\right) \Delta s\right], \quad t \in I
$$

Remark 3.2 If we take $a(t) \equiv u_{0}, g(s, t)=g(t)$ and $\lambda=0$, then Theorem 3.3 reduces to [27, Theorem 1 ]. If we take $\mathbb{T}=\mathbb{R}, a(t) \equiv u_{0}$ and $\lambda=0$, then Theorem 3.3 reduces to [32, Theorem $2.1\left(a_{1}\right)$ ]. If we take $\mathbb{T}=\mathbb{Z}, a(t) \equiv u_{0}$ and $\lambda=0$, then Theorem 3.3 reduces to [32, Theorem $\left.2.3\left(c_{1}\right)\right]$.

\section{Applications}

In this section, we will present some simple applications for our results. 
Example 4.1 Consider the following retarded Volterra-Fredholm type dynamic integral equation on time scales:

$$
\begin{aligned}
u(t)= & a(t)+b(t) \int_{\alpha\left(t_{0}\right)}^{\alpha(t)} F\left(s, u(s), \int_{\alpha\left(t_{0}\right)}^{s} H(\tau, u(\tau)) \Delta \tau\right) \Delta s \\
& +\int_{\alpha\left(t_{0}\right)}^{\alpha(T)} \widetilde{F}\left(s, u(s), \int_{\alpha\left(t_{0}\right)}^{s} \widetilde{H}(\tau, u(\tau)) \Delta \tau\right) \Delta s, \quad t \in I,
\end{aligned}
$$

where $u, a, b: I \rightarrow \mathbb{R}$ are rd-continuous functions, $|a|$ is nondecreasing, $b(T) \neq 0, \alpha \in \Upsilon$, $F, \widetilde{F}: I \times \mathbb{R} \times \mathbb{R} \rightarrow \mathbb{R}$ and $H, \widetilde{H}: I \times \mathbb{R} \rightarrow \mathbb{R}$ are continuous functions.

The following theorem gives an estimate for the solutions of Eq. (4.1).

Theorem 4.1 Suppose that the functions $F, H, \widetilde{F}$ and $\widetilde{H}$ in (4.1) satisfy the conditions

$$
\begin{aligned}
& |F(t, u, v)| \leq f_{1}(t)|u|+f_{2}(t)|v|, \quad t \in I, u, v \in \mathbb{R} \\
& |H(t, u)| \leq g(t)|u|, \quad t \in I, u \in \mathbb{R} \\
& |\widetilde{F}(t, u, v)| \leq f_{1}(t)|u|+f_{2}(t)|v|, \quad t \in I, u, v \in \mathbb{R} \\
& |\widetilde{H}(t, u)| \leq g(t)|u|, \quad t \in I, u \in \mathbb{R}
\end{aligned}
$$

where $f_{1}, f_{2}, g: I \rightarrow \mathbb{R}_{+}$are rd-continuous functions. If

$$
\beta:=e_{B \oplus C}\left(T, t_{0}\right)<1+|b(T)|,
$$

then all solutions of Eq. (4.1) satisfy

$$
|u(t)| \leq \frac{|a(T) b(T)|}{1+|b(T)|-\beta} e_{B \oplus C}\left(t, t_{0}\right), \quad t \in I,
$$

where $B(t)$ and $C(t)$ are defined as in Theorem 3.1.

Proof By (4.1)-(4.3), we get

$$
\begin{aligned}
|u(t)| \leq & |a(t)|+|b(t)| \int_{\alpha\left(t_{0}\right)}^{\alpha(t)}\left|F\left(s, u(s), \int_{\alpha\left(t_{0}\right)}^{s} H(\tau, u(\tau)) \Delta \tau\right)\right| \Delta s \\
& +\int_{\alpha\left(t_{0}\right)}^{\alpha(T)}\left|\widetilde{F}\left(s, u(s), \int_{\alpha\left(t_{0}\right)}^{s} \widetilde{H}(\tau, u(\tau)) \Delta \tau\right)\right| \Delta s \\
\leq & |a(t)|+|b(t)| \int_{\alpha\left(t_{0}\right)}^{\alpha(t)}\left[f_{1}(s)|u(s)|+f_{2}(s) \int_{\alpha\left(t_{0}\right)}^{s} g(\tau)|u(\tau)| \Delta \tau\right] \Delta s \\
& +\int_{\alpha\left(t_{0}\right)}^{\alpha(T)}\left[f_{1}(s)|u(s)|+f_{2}(s) \int_{\alpha\left(t_{0}\right)}^{s} g(\tau)|u(\tau)| \Delta \tau\right] \Delta s, \quad t \in I .
\end{aligned}
$$

Using Corollary 3.1, we obtain the desired inequality (4.4).

The next result deals with the uniqueness of solutions of Eq. (4.1). 
Theorem 4.2 Suppose that the functions $F, H, \widetilde{F}$ and $\widetilde{H}$ in (4.1) satisfy the conditions

$$
\begin{aligned}
& \left|F\left(t, u_{1}, v_{1}\right)-F\left(t, u_{2}, v_{2}\right)\right| \leq f_{1}(t)\left|u_{1}-u_{2}\right|+f_{2}(t)\left|v_{1}-v_{2}\right|, \quad t \in I, u, v \in \mathbb{R}, \\
& \left|H\left(t, u_{1}\right)-H\left(t, u_{2}\right)\right| \leq g(t)\left|u_{1}-u_{2}\right|, \quad t \in I, u_{1}, u_{2} \in \mathbb{R}, \\
& \left|\widetilde{F}\left(t, u_{1}, v_{1}\right)-\widetilde{F}\left(t, u_{2}, v_{2}\right)\right| \leq f_{1}(t)\left|u_{1}-u_{2}\right|+f_{2}(t)\left|v_{1}-v_{2}\right|, \quad t \in I, u, v \in \mathbb{R}, \\
& \left|\widetilde{H}\left(t, u_{1}\right)-\widetilde{H}\left(t, u_{2}\right)\right| \leq g(t)\left|u_{1}-u_{2}\right|, \quad t \in I, u_{1}, u_{2} \in \mathbb{R},
\end{aligned}
$$

where $f_{1}, f_{2}, g: I \rightarrow \mathbb{R}_{+}$are rd-continuous functions. If

$$
\beta:=e_{B \oplus C}\left(T, t_{0}\right)<1+|b(T)|,
$$

then Eq. (4.1) has at most one solution on I, where $B(t)$ and $C(t)$ are defined as in Theorem 3.1 .

Proof Let $u(t)$ and $v(t)$ be two solutions of Eq. (4.1) on I. From (4.1) and (4.7)-(4.10), we have

$$
\begin{aligned}
& |u(t)-v(t)| \\
& \leq|b(t)| \int_{\alpha\left(t_{0}\right)}^{\alpha(t)}\left[f_{1}(s)|u(s)-v(s)|+f_{2}(s) \int_{\alpha\left(t_{0}\right)}^{s} g(\tau)|u(\tau)-v(\tau)| \Delta \tau\right] \Delta s \\
& \quad+\int_{\alpha\left(t_{0}\right)}^{\alpha(T)}\left[f_{1}(s)|u(s)-v(s)|+f_{2}(s) \int_{\alpha\left(t_{0}\right)}^{s} g(\tau)|u(\tau)-v(\tau)| \Delta \tau\right] \Delta s, \quad t \in I .
\end{aligned}
$$

Applying Corollary 3.1 to (4.11), we get $|u(t)-v(t)| \leq 0, t \in I$. Therefore $u(t) \equiv v(t)$, that is, there is at most one solution to Eq. (4.1).

Example 4.2 Assume $\mathbb{T}=q^{\mathbb{N}_{0}}=\left\{1, q, q^{2}, \ldots\right\}$ with $q>1$ and consider the following Volterra-Fredholm type $q$-difference equation:

$$
\begin{aligned}
u(t)= & a(t)+b(t) \sum_{s=t_{0}}^{t}\left[f_{1}(s) u(s)+f_{2}(s) \sum_{\tau=t_{0}}^{s} g(\tau) u(\tau)\right] \\
& +\sum_{s=t_{0}}^{T}\left[f_{1}(s) u(s)+f_{2}(s) \sum_{\tau=t_{0}}^{s} g(\tau) u(\tau)\right], \quad t \in I,
\end{aligned}
$$

where $u, a, b, f_{1}, f_{2}, g: I \rightarrow \mathbb{R}_{+}$are functions, $t_{0}, T \in q^{\mathbb{N}_{0}}, T>t_{0}$ and $I=\left[t_{0}, T\right] \cap q^{\mathbb{N}_{0}}$.

Theorem 4.3 Assume that a is nondecreasing and $(q-1) t A(t)<1$. If

$$
\beta:=\prod_{\tau=t_{0}}^{T / q}[1+(q-1) \tau(B(\tau)+C(\tau)+(q-1) \tau B(\tau) C(\tau))]<1+b(T),
$$

then the solution $u(t)$ of Eq. (4.12) satisfies the following inequality:

$$
u(t) \leq \frac{a(T) b(T)}{1+b(T)-\beta} \prod_{\tau=t_{0}}^{t / q}[1+(q-1) \tau(B(\tau)+C(\tau)+(q-1) \tau B(\tau) C(\tau))], \quad t \in I,
$$


where

$$
\begin{aligned}
& A(t):=\frac{b(q t)-b(t)}{(q-1) t} \sum_{s=t_{0}}^{q t}\left[f_{1}(s)+f_{2}(s) \sum_{\tau=t_{0}}^{s} g(\tau)\right], \quad B(t):=\frac{A(t)}{1-(q-1) t A(t)}, \\
& C(t):=b(t)\left[f_{1}(t)+f_{2}(t) \sum_{\tau=t_{0}}^{t} g(\tau)\right] .
\end{aligned}
$$

Proof Note that $\sigma(t)=q t, \mu(t)=(q-1) t$ for any $t \in q^{\mathbb{N}_{0}}$ and

$$
e_{p}\left(t, t_{0}\right)=\prod_{\tau=t_{0}}^{t / q}[1+(q-1) \tau p(\tau)]
$$

for $t>t_{0}$, where $t, t_{0}, \tau \in q^{\mathbb{N}_{0}}$. Let $u(t)$ be a solution of Eq. (4.12), we get

$$
\begin{aligned}
|u(t)| \leq & a(t)+b(t) \sum_{s=t_{0}}^{t}\left[f_{1}(s)|u(s)|+f_{2}(s) \sum_{\tau=t_{0}}^{s} g(\tau)|u(\tau)|\right] \\
& +\sum_{s=t_{0}}^{T}\left[f_{1}(s)|u(s)|+f_{2}(s) \sum_{\tau=t_{0}}^{s} g(\tau)|u(\tau)|\right], \quad t \in I .
\end{aligned}
$$

Then a suitable application of Corollary 3.1 to (4.14) yields the desired result (4.13).

Example 4.3 Assume $\mathbb{T}=\mathbb{R}$ and consider the following retarded Volterra-Fredholm type integral equation:

$$
\begin{aligned}
u(t)= & a(t)+b(t) \int_{\alpha\left(t_{0}\right)}^{\alpha(t)}\left[f_{1}(s) u(s)+f_{2}(s) \int_{\alpha\left(t_{0}\right)}^{s} g(\tau) u(\tau) \mathrm{d} \tau\right] \mathrm{d} s \\
& +\lambda \int_{\alpha\left(t_{0}\right)}^{\alpha(T)}\left[f_{1}(s) u(s)+f_{2}(s) \int_{\alpha\left(t_{0}\right)}^{s} g(\tau) u(\tau) \mathrm{d} \tau\right] \mathrm{d} s, \quad t \in I,
\end{aligned}
$$

where $u, a, b, \alpha, f_{1}, f_{2}, g: I \rightarrow \mathbb{R}_{+}$are continuous functions, $t_{0}, T \in \mathbb{R}, T>t_{0}$ and $I=\left[t_{0}, T\right]$.

Theorem 4.4 Assume that $a$ is nondecreasing, $\lambda \geq 0$ is a constant, $\alpha^{\prime}(t)>0, \alpha(t) \leq t$ and $b^{\prime}(t) \geq 0$. If

$$
\beta:=\exp \left(\int_{t_{0}}^{T}(A+C)(\tau) \mathrm{d} \tau\right)<1+\frac{b(T)}{\lambda},
$$

then the solution $u(t)$ of Eq. (4.15) satisfies the following inequality:

$$
u(t) \leq \frac{a(T) b(T)}{\lambda+b(T)-\beta \lambda} \exp \left(\int_{t_{0}}^{t}(A+C)(\tau) \mathrm{d} \tau\right), \quad t \in I,
$$


where we use the convention that $\frac{1}{0}=+\infty$,

$$
\begin{aligned}
& A(t):=b^{\prime}(t) \int_{\alpha\left(t_{0}\right)}^{\alpha(t)}\left[f_{1}(s)+f_{2}(s) \int_{\alpha\left(t_{0}\right)}^{s} g(\tau) \mathrm{d} \tau\right] \mathrm{d} s \text { and } \\
& C(t):=b(t)\left[f_{1}(\alpha(t))+f_{2}(\alpha(t)) \int_{\alpha\left(t_{0}\right)}^{\alpha(t)} g(\tau) \mathrm{d} \tau\right] \alpha^{\prime}(t) .
\end{aligned}
$$

Proof Note that $\sigma(t)=t, \mu(t)=0$ for any $t \in \mathbb{R}$, and

$$
e_{p}\left(t, t_{0}\right)=\exp \left(\int_{t_{0}}^{t} p(\tau) \mathrm{d} \tau\right)
$$

for $t>t_{0}$. Let $u(t)$ be a solution of Eq. (4.15), we have

$$
\begin{aligned}
|u(t)| \leq & a(t)+b(t) \int_{\alpha\left(t_{0}\right)}^{\alpha(t)}\left[f_{1}(s)|u(s)|+f_{2}(s) \int_{\alpha\left(t_{0}\right)}^{s} g(\tau)|u(\tau)| \mathrm{d} \tau\right] \mathrm{d} s \\
& +\lambda \int_{\alpha\left(t_{0}\right)}^{\alpha(T)}\left[f_{1}(s)|u(s)|+f_{2}(s) \int_{\alpha\left(t_{0}\right)}^{s} g(\tau)|u(\tau)| \mathrm{d} \tau\right] \mathrm{d} s, \quad t \in I .
\end{aligned}
$$

Then a suitable application of Theorem 3.1 to (4.17) yields the desired result (4.16).

\section{Conclusions}

In this paper we have established some new retarded Volterra-Fredholm type integral inequalities on time scales. Unlike some existing results in the literature (e.g., [16, 20]), the integral inequalities considered in this paper involve the retarded term, which results in difficulties in the estimation on the explicit bounds of unknown functions $u(t)$. These inequalities generalize and extend some known inequalities and can be used as tools in the qualitative theory of certain classes of retarded dynamic equations on time scales.

\section{Acknowledgements}

The author thanks the reviewers for their helpful and valuable suggestions and comments on this paper. This research was supported by the National Natural Science Foundation of China (No. 11671227) and the Project of Shandong Province Higher Educational Science and Technology Program (China) (No. J14LI09).

\section{Competing interests}

The author declares that there is no conflict of interests regarding the publication of this paper.

\section{Authors' contributions}

The author read and approved the final manuscript.

\section{Publisher's Note}

Springer Nature remains neutral with regard to jurisdictional claims in published maps and institutional affiliations.

Received: 2 August 2017 Accepted: 13 November 2017 Published online: 28 November 2017

References

1. Hilger, S: Ein Maßkettenkalkül mit Anwendung auf Zentrumsmanningfaltigkeiten. PhD thesis, Universität Würzburg (1988)

2. Adivar, M, Raffoul, YN: Existence results for periodic solutions of integro-dynamic equations on time scales. Ann. Mat. Pura Appl. 188, 543-559 (2009)

3. Atici, FM, Biles, DC, Lebedinsky, A: An application of time scales to economics. Math. Comput. Model. 43, 718-726 (2006)

4. $\mathrm{Bi}, \mathrm{L}$, Bohner, M, Fan, M: Periodic solutions of functional dynamic equations with infinite delay. Nonlinear Anal. 68 , 170-174 (2008) 
5. Dogan, A, Graef, J, Kong, L: Higher-order singular multi-point boundary-value problems on time scales. Proc. Edinb. Math. Soc. 54, 345-361 (2011)

6. Erbe, L, Jia, BG, Peterson, A: Belohorec-type oscillation theorem for second order sublinear dynamic equations on time scales. Math. Nachr. 284, 1658-1668 (2011)

7. Erbe, L, Jia, BG, Peterson, A: On the asymptotic behavior of solutions of Emden-Fowler equations on time scales. Ann. Mat. Pura Appl. 191, 205-217 (2012)

8. Federson, M, Mesquita, JG, Slavik, A: Measure functional differential equations and functional dynamic equations on time scales. J. Differ. Equ. 252, 3816-3847 (2012)

9. Karpuz, B: Volterra theory on time scales. Results Math. 65, 263-292 (2014)

10. Slavik, A: Averaging dynamic equations on time scales. J. Math. Anal. Appl. 388, 996-1012 (2012)

11. Agarwal, R, Bohner, M, Peterson, A: Inequalities on time scales: a survey. Math. Inequal. Appl. 4, 535-557 (2001)

12. Anderson, DR: Nonlinear dynamic integral inequalities in two independent variables on time scale pairs. Adv. Dyn. Syst. Appl. 3, 1-13 (2008)

13. Bohner, EA, Bohner, M, Akin, F: Pachpatte inequalities on time scale. J. Inequal. Pure Appl. Math. 6(1), Article ID 6 (2005)

14. Feng, Q, Meng, F, Zheng, B: Gronwall-Bellman type nonlinear delay integral inequalities on time scale. J. Math. Anal. Appl. 382, 772-784 (2011)

15. Feng, Q, Zheng, B: Generalized Gronwall-Bellman-type delay dynamic inequalities on time scales and their applications. Appl. Math. Comput. 218, 7880-7892 (2012)

16. Gu, J, Meng, F: Some new nonlinear Volterra-Fredholm type dynamic integral inequalities on time scales. Appl. Math. Comput. 245, 235-242 (2014)

17. Li, WN: Some delay integral inequalities on time scales. Comput. Math. Appl. 59, 1929-1936 (2010)

18. $\mathrm{Ma}, \mathrm{QH}$, Pečarić, J: The bounds on the solutions of certain two-dimensional delay dynamic systems on time scales. Comput. Math. Appl. 61, 2158-2163 (2011)

19. $\mathrm{Ma}, \mathrm{QH}$, Wang, JW, Ke, XH, Pečarić, J: On the boundedness of a class of nonlinear dynamic equations of second order. Appl. Math. Lett. 26(11), 1099-1105 (2013)

20. Meng, F, Shao, J: Some new Volterra-Fredholm type dynamic integral inequalities on time scales. Appl. Math. Comput. 223, 444-451 (2013)

21. Pachpatte, DB: Explicit estimates on integral inequalities with time scale. J. Inequal. Pure Appl. Math. 7, Article ID 143 (2006)

22. Saker, SH: Some nonlinear dynamic inequalities on time scales and applications. J. Math. Inequal. 4, 561-579 (2010)

23. Saker, SH: Nonlinear dynamic inequalities of Gronwall-Bellman type on time scales. Electron. J. Qual. Theory Differ. Equ. 2011, 86 (2011)

24. Saker, SH: Some nonlinear dynamic inequalities on time scales. Math. Inequal. Appl. 14, 633-645 (2011)

25. Sun, YG: Some sublinear dynamic integral inequalities on time scales. Math. Inequal. Appl. 15, 331-341 (2012)

26. Sun, YG, Hassan, T: Some nonlinear dynamic integral inequalities on time scales. Appl. Math. Comput. 220, 221-225 (2013)

27. Wong, F, Yeh, CC, Hong, CH: Gronwall inequalities on time scales. Math. Inequal. Appl. 9, 75-86 (2006)

28. Wong, FH, Yu, SL, Yeh, CC: Anderson's inequality on time scales. Appl. Math. Lett. 19, 931-935 (2006)

29. Yin, L, Luo, QM, Qi, F: Several integral inequalities on time scales. J. Math. Inequal. 6, 419-429 (2012)

30. Bohner, M, Peterson, A: Dynamic Equations on Time Scales: An Introduction with Applications. Birkhäuser, Boston (2001)

31. Bohner, M, Peterson, A: Advances in Dynamic Equations on Time Scales. Birkhäuser, Boston (2003)

32. Pachpatte, BG: Bounds on certain integral inequalities. J. Inequal. Pure Appl. Math. 3, Article ID 47 (2002)

\section{Submit your manuscript to a SpringerOpen ${ }^{\circ}$ journal and benefit from:}

- Convenient online submission

- Rigorous peer review

- Open access: articles freely available online

- High visibility within the field

- Retaining the copyright to your article

Submit your next manuscript at $\gg$ springeropen.com 